\title{
ESTUDOS TERMOANALÍTICOS DE SAIS DE SÓDIO E POTÁSSIO DE DITIOCARBAMATOS EM ATMOSFERA OXIDANTE
}

\author{
Luiz Antônio Ramos, Éder Tadeu Gomes Cavalheiro* e Gilberto Orivaldo Chierice \\ Instituto de Química de São Carlos, Universidade de São Paulo, Av. do Trabalhador São-Carlense, 400, 13566-950 \\ São Carlos - SP, Brasil
}

Recebido em 5/12/07; aceito em 11/4/08; publicado na web em 19/9/08

\begin{abstract}
THERMOANALYTICAL STUDIES OF DITHIOCARBAMATE OF SODIUM AND POTASSIUM SALTS IN AN OXIDIZING ATMOSPHERE. Thermoanalytical behavior of sodium and potassium salts of pyrrolidinedithiocarbamate (pyr), piperidineditiocarbamate (pip), morpholinedithiocarbamate (mor), hexametileneiminedithiocarbamate (hex), were investigated. In a first step the salts were synthesized and characterized by infrared spectroscopy (FTIR), ${ }^{1} \mathrm{H}$ and ${ }^{13} \mathrm{C}$ nuclear magnetic resonance (NMR) and elementar analysis. Finally, thermal analytical (TG/DTG and DSC) studies were performed in order to evaluate the thermal stability, as well as the pathways of the thermal decomposition based in the intermediate and final decomposition products.
\end{abstract}

Keywords: dithiocarbamic acid; thermal behavior; themal analytical.

\section{INTRODUÇÃO}

Os ditiocarbamatos (DTC) são produtos da reação entre uma amina primária ou secundária com o dissulfeto de carbono, em geral na presença de uma base. São estudados desde 1850, quando a primeira síntese foi registrada. ${ }^{1}$ Dependendo do tipo de amina usada na síntese pode ser obtido um mono ou um diaquilditiocarbamato ou um derivado cíclico.

Os DTC's têm sido estudados extensivamente nas últimas décadas, devido à vasta gama de aplicação em diferentes áreas, como na química, indústria e medicina..$^{2-4}$

Também são muito utilizados na agricultura como inseticidas, herbicidas e fungicidas. Alguns são usados no controle de vetor em saúde pública. ${ }^{5}$ Estudos termoanalíticos de complexos de metais com ditiocarbamatos são extensamente descritos na literatura. ${ }^{6-10}$

Entretanto, apesar da importância como intermediários de reação e precursores de complexos metálicos, os sais de sódio e potássio de ditiocarbamatos são raramente descritos na literatura em relação às propriedades térmicas, ${ }^{11-14}$ especialmente no caso dos sais de potássio, cuja síntese é difícil devido à sua elevada solubilidade em meio aquoso. A purificação deste sal pode ser obtida por recristalização, mas os rendimentos costumam ser muito baixos, também devido à sua alta solubilidade, assim, pode ocorrer a decomposição do sal sintetizado. Os procedimentos e os rendimentos destas sínteses não são, geralmente, relatados na literatura, ao menos de maneira sistemática.

Assim, a busca de novas rotas sintéticas mais simples e com bons rendimentos de reação pode representar uma importante contribuição à preparação destes compostos. ${ }^{15,16}$

Os sais de sódio e potássio são costumeiramente usados na preparação de complexos de ditiocarbamatos com íons metálicos, cujo comportamento térmico foi descrito previamente. ${ }^{17-23}$ Considerando a relevância e os poucos dados descritos na literatura, o presente trabalho trata da síntese e caracterização, assim como do estudo termoanalíticos envolvendo TG/DTG e DSC de sais de $\mathrm{Na}\left[\mathrm{S}_{2} \mathrm{CNR}\right]$ e $\mathrm{K}\left[\mathrm{S}_{2} \mathrm{CNR}\right]$ derivados de aminas cíclicas ( $\mathrm{R}=$ pirrolidina (pyr), piperidina (pip), morfolina (mor), hexametileno (hex)), em atmosfera de ar.

\footnotetext{
*e-mail: cavalheiro@iqsc.usp.br
}

\section{PARTE EXPERIMENTAL}

\section{Síntese e caracterização dos sais de DTC}

Os sais de sódio de DTC foram preparados pela reação direta entre amina e dissulfeto de carbono $\left(\mathrm{CS}_{2}\right)$, na presença de uma quantidade estequiométrica de hidróxido de sódio em álcool etílico/água 1:1 ( $\left(\mathrm{v}_{\mathrm{v}}\right)$. A mistura reacional foi colocada no freezer por $12 \mathrm{~h} \mathrm{e,} \mathrm{em} \mathrm{seguida,}$ filtrada em funil de Büchner, lavada com éter gelado e o produto recristalizado em uma mistura etanol água $1: 1(\mathrm{v} / \mathrm{v}) .{ }^{14,22}$

Os sais de potássio de DTC foram preparados de acordo com o procedimento previamente descrito. ${ }^{17}$ Por se tratar de sais com solubilidade elevada, foi necessário fazer um estudo do meio reacional para se otimizar uma rota sintética, que fornecesse um bom rendimento. Os dois procedimentos que apresentaram os melhores rendimentos para os sais potássio, entre vários testados, foram:

\section{Procedimento 1}

Em um erlenmeyer de $250 \mathrm{~mL}$, com tampa de vidro esmerilhado, foi colocado um volume correspondente a $0,10 \mathrm{~mol} \mathrm{~L}^{-1}$ da amina desejada, o qual foi diluído com uma mistura de etanol/água 3:1 (v/ $)$, em quantidade igual a 3 vezes o volume da amina. A esta mistura juntou-se $0,10 \mathrm{~mol} \mathrm{~L}^{-1}$ de hidróxido de potássio (Merck). Esta solução foi colocada em banho de gelo e sal. Após resfriamento adicionouse $0,10 \mathrm{~mol} \mathrm{~L}^{-1}$ de dissulfeto de carbono (Carlo Erba), gota a gota e com agitação constante.

A mistura reacional foi colocada no freezer por $12 \mathrm{~h}$, ocorrendo a precipitação de uma pequena quantidade dos sais, insuficiente para recristalização. Este método apresentou um rendimento muito baixo, da ordem de $15 \%$.

Outros solventes foram testados, como álcool etílico anidro (Merck), álcool metílico (Carlo Erba), acetona (Carlo Erba), éter etílico (Merck) e álcool isopropílico (Merck); entretanto, o rendimento também foi baixo, em torno de $15 \%$.

\section{Procedimento 2}

Em um erlenmeyer com tampa de vidro esmerilhado, foram colocados 0,10 $\mathrm{mol} \mathrm{L}^{-1}$ de hidróxido de potássio (Merck), 5,0 mL de água deionizada e 25,0 mL de álcool isopropílico (Merck), deixando- 
se sob agitação até dissolução total do hidróxido. Em seguida foram adicionados $0,10 \mathrm{~mol} \mathrm{~L}^{-1}$ da amina desejada. Esta solução foi colocada em banho de gelo e sal. Após resfriamento foram adicionados 0,10 $\mathrm{mol} \mathrm{L}^{-1}$ de dissulfeto de carbono (Carlo Erba), gota a gota sob agitação constante.

Após 2 h de agitação constante, a mistura reacional foi colocada no freezer por $12 \mathrm{~h}$ e, em seguida, o precipitado foi filtrado em funil de Büchner, lavado com éter gelado e recristalizado em uma mistura isopropanol/água 1:5 (v/v).

Com este procedimento obtiveram-se rendimentos para os sais de potássio da ordem de 40 a $60 \%$. Apesar de várias tentativas não foi possível obter o Khex, sendo sempre o Hhexhex - hexametilenoditiocarbamato da hexametilenoimina protonada o produto final nestas sínteses.

$\mathrm{Na}$ caracterização foram utilizadas análise elementar $(\mathrm{C}, \mathrm{N}, \mathrm{H})$, espectroscopia na região do infravermelho (FTIR) e espectrometria de $\mathrm{RMN}$ de ${ }^{1} \mathrm{He}{ }^{13} \mathrm{C}$.

As análises elementares foram realizadas em um analisador CHNS-O da Fisons Instruments, EA 1108.

Os espectros de FTIR foram obtidos em um espectrofotômetro da Bomem Michelson, MB-102-FTIR na região entre 4000 e 400 $\mathrm{cm}^{-1}$, com as amostras sendo diluídas em CsI (Merck) 1/100 (m/ $)$ e prensadas na forma de pastilhas. O CsI foi mantido em estufa a 110 ${ }^{\circ} \mathrm{C}$, antes de ser utilizado.

Os espectros de $\mathrm{RMN}$ de ${ }^{1} \mathrm{H}$ e ${ }^{13} \mathrm{C}$ foram obtidos em um espectrofotômetro da Brüker Arx, com frequiência de $200 \mathrm{MHz}$, utilizando soluções de água deuterada, na qual dissolveram-se aproximadamente $5 \mathrm{mg}$ de cada amostra, para cada $100 \mu \mathrm{L}$.

\section{Estudos termoanalíticos com sais de DTC}

As curvas termogravimétricas foram obtidas em módulo Termogravimétrico TG-951, acoplado a um analisador térmico TA-2000 (ambos da TA Instruments) e equipado com registrador HP7440A.

Nos estudos termogravimétricos foram usadas massas das amostras da ordem de 7,0 mg, com precisão de $\pm 1 \mu \mathrm{g}$. Nos estudos para definição dos melhores parâmetros experimentais, utilizou-se razão de aquecimento de $5,10,15$ e $20^{\circ} \mathrm{C} \mathrm{min}^{-1}$, sob atmosfera dinâmica de ar, com vazão de gás de $25,50,75$ e $100 \mathrm{~mL} \mathrm{~min}^{-1}$, em suporte de amostra de platina. A temperatura máxima foi de $900{ }^{\circ} \mathrm{C}$. A razão de aquecimento escolhida foi de $10{ }^{\circ} \mathrm{C} \min ^{-1}$ e a vazão de $100 \mathrm{~mL}$ $\min ^{-1}$.

As medidas calorimétricas foram realizadas em um módulo calorimétrico exploratório diferencial DSC-910, acoplado a um analisador térmico TA-2000 (ambos da TA Instruments) e equipado com registrador HP7440A.

As curvas DSC foram obtidas utilizando massas de amostra em torno de 3,0 mg com precisão de $\pm 0,1 \mathrm{mg}$, em suporte de amostra de alumínio tampado, com furo na parte central superior. A razão de aquecimento foi de $5{ }^{\circ} \mathrm{C} \mathrm{min}^{-1}$, sob atmosfera dinâmica de ar com vazão de $100 \mathrm{~mL} \mathrm{~min}^{-1}$. A temperatura máxima foi de $500{ }^{\circ} \mathrm{C}$.

\section{RESULTADOS E DISCUSSÃO}

A representação das estruturas dos ligantes sintetizados é apresentada na Tabela 1. Os resultados para \% C, N, H experimental (calculado), para os sais foram, respectivamente, Napyr.2H $\mathbf{O}: 29,15$ $(29,26) ; 6,78$ (6,82); 5,90(5,89); Kpyr.0,25H_O: $31,83^{2}(32,40)$; 7,83 (7,56); 4,22 (4,35); Napip.2H $\mathbf{O}: 33,10(32,86) ; 6,45(6,39)$; 6,48 (6,43); Крip.2H $\mathbf{O}$ : 30,62 (30,61); 5,97 (5,95); 6,07 (5,99); Namor.2H_O: 27,65 (27,14); 6,46 (6,33); 5,64 (5,47); Kmor.H_O: $27,53(27,38) ; 6,42(6,39) ; 4,47$ (4,59); Nahex.2H_O: 56,78 (56,89); $10,30(10,21) ; 9,43(9,55)$ e sugerem diferenças na quantidade de moléculas de água de hidratação.

Segundo Nakamoto, ${ }^{24}$ as freqüências do estiramento da ligação carbono-nitrogênio $v(\mathrm{C}-\mathrm{N})$ apresentam absorção intensa na região entre 1600-1450 $\mathrm{cm}^{-1}$. As bandas do estiramento da ligação carbonoenxofre ( $\vee C-S$ ) são representadas por uma ou duas bandas com absorção intensa na região de $900-1000 \mathrm{~cm}^{-1}$ e sugerem uma coordenação bidentada ou nos sais, quando há apenas uma banda, e monodentada, quando há duas bandas.

Os compostos preparados neste trabalho apresentaram as seguintes absorções para os estiramentos $v(\mathrm{C}-\mathrm{N})$ e (vC-S): Napyr: $1461 \mathrm{e}$ 942/998; Napyr: 1461 e 942/998; Kpyr 1461 e 947/1004; Napip: 1470 e 942/999; Kpip: 1468 e 968/1005; Namor: 1463 e 982/1025; Kmor: 1450 e 975/1012; Nahex: 1485 e 975/1010; o dublete na segunda banda sugere que todos são sais. ${ }^{24}$

Na Tabela 1 estão os valores do deslocamento químico $(\delta)$, em ppm observados na espectrometria de $\mathrm{RMN}$ de ${ }^{13} \mathrm{C}-\left(\mathrm{D}_{2} \mathrm{O}\right)$ e ${ }^{1} \mathrm{H}-\left(\mathrm{D}_{2} \mathrm{O}\right)$ dos sais de sódio e potássio. Observou-se que o deslocamento químico do átomo de carbono no fragmento $\mathrm{NCS}_{2}$ nos sais de DTC apresentou

Tabela 1. Deslocamentos químicos da série de sais obtidos por espectrometria de $\mathrm{RMN}$ de ${ }^{13} \mathrm{C}-\left(\mathrm{D}_{2} \mathrm{O}\right)$ e ${ }^{1} \mathrm{H}-\left(\mathrm{D}_{2} \mathrm{O}\right)^{*}$

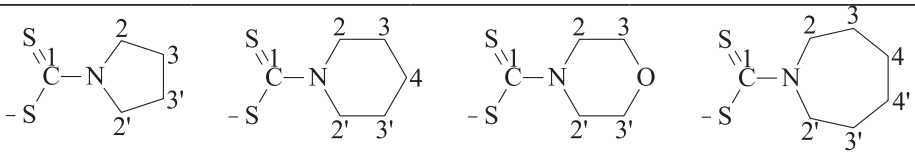

pyr pip hor hex

\begin{tabular}{|c|c|c|c|c|c|c|c|}
\hline \multirow{2}{*}{ Composto } & \multicolumn{3}{|c|}{${ }^{13} \mathrm{C}-\left(\mathrm{D}_{2} \mathrm{O}\right) \mathrm{ppm}$} & \multirow[b]{2}{*}{$\delta_{4}$} & \multicolumn{3}{|c|}{${ }^{1} \mathrm{H}-\left(\mathrm{D}_{2} \mathrm{O}\right) \mathrm{ppm}$} \\
\hline & $\delta_{1}$ & $\delta_{2}$ & $\delta_{3}$ & & $\delta_{2}$ & $\delta_{3}$ & $\delta_{4}$ \\
\hline Napyr & 193,37 & 45,83 & 16,52 & --- & 3,75 & 1,96 & -- \\
\hline Kpyr & 194,02 & 45,73 & 16,40 & --- & 3,73 & 1,96 & --- \\
\hline Napip & 196,02 & 43,89 & 16,52 & 14,66 & 4,21 & 1,56 & 1,53 \\
\hline Kpip & 196,02 & 43,85 & 16,66 & 14,82 & 4,19 & 1,56 & 1,53 \\
\hline Namor & 200,19 & 42,38 & 57,16 & --- & 4,32 & 3,75 & --- \\
\hline Kmor & 200,13 & 42,12 & 56,87 & --- & 4,36 & 3,74 & --- \\
\hline Nahex & 197,64 & 46,33 & 17,64 & 17,23 & 4,13 & 1,73 & 1,53 \\
\hline
\end{tabular}

$*$ pyr $=$ pirrolidinaditiocarbamato, pip $=$ piperidinaditiocarbamato, mor = morfolinaditiocarbamato, hex $=$ hexametilenoiminaditiocarbamato 
um deslocamento pouco significativo para os diferentes contra-íons e também se observou uma pequena variação, naquele parâmetro, com as mudanças na estrutura molecular no caso do aumento do anel.

Esta pequena variação no deslocamento químico no fragmento $\mathrm{NCS}_{2}$ dos sais DTC pode estar relacionada com a análise dos sais livres em solução, uma vez que se tratam de compostos iônicos. Para os sais livres não há nenhuma compensação da carga nos átomos de enxofre por átomos coordenados ocasionando, assim, um efeito muito pequeno no deslocamento químico no fragmento $-\mathrm{NCS}_{2}$.

\section{Resultados termoanalíticos dos sais de sódio}

As perdas de massa, atribuídas aos eventos térmicos observados nas curvas TG/DTG e DSC, e os intervalos de temperatura para os sais de sódio estão reunidos na Tabela 2.

A Figura 1 representa as curvas TG/DTG, enquanto a Figura 2 representa as curvas DSC obtidas em atmosfera dinâmica de ar com vazão de $100 \mathrm{~mL} \mathrm{~min}{ }^{-1}$ e razão de aquecimento de $10{ }^{\circ} \mathrm{C}$ min $^{-1}$.

A partir destas Figuras e da Tabela 2 é possível observar que os sais de Napyr, Napip, Namor e Nahex apresentam um processo de desidratação no intervalo de temperatura entre 50 e $150^{\circ} \mathrm{C}$, com perdas de massa características para cada caso. Em seguida ocorre decomposição, gerando como resíduo o sulfato de sódio, o que está de acordo com os valores dos cálculos estequiométricos para $1 \frac{1}{2} \mathrm{de} \mathrm{Na}_{2} \mathrm{SO}_{4}$ e com os espectros de FTIR dos resíduos em comparação com o espectro do $\mathrm{Na}_{2} \mathrm{SO}_{4}$.

Os eventos térmicos presentes nas curvas DSC (Figura 2) mostraram-se coerentes com as observações das curvas TG/DTG quanto aos processos que envolvem perdas de massa.

Observaram-se processos de desidratação acompanhada da decomposição para Napyr, Napip e Namor. Já para o Nahex, a curva DSC apresentou uma sequiência de picos endotérmicos atribuídos à desidratação, sendo o último a fusão, seguida de um pico exotérmico atribuído à decomposição. Isto sugere um mecanismo diferenciado para desidratação do Nahex, já que a perda de $\mathrm{H}_{2} \mathrm{O}$ ocorre em duas etapas e a presença do processo de fusão, que também o diferencia dos demais.

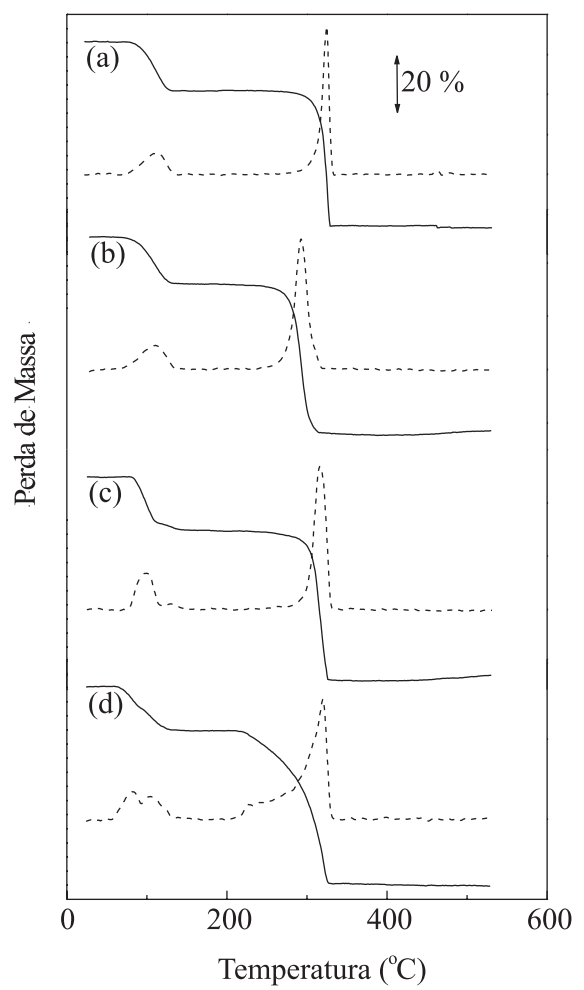

Figura 1. Curvas TG (sólido) e DTG (pontilhado) para os sais: (a) Napyr.2 $\mathrm{H}_{2} \mathrm{O}$; (b) Napip. $2 \mathrm{H}_{2} \mathrm{O}$; (c) Namor. $2 \mathrm{H}_{2} \mathrm{O}$ e (d) Nahex. $2 \mathrm{H}_{2} \mathrm{O}$, sob as condições descritas no texto

\section{Resultados termoanalíticos dos sais de potássio}

As perdas de massa, atribuídas aos eventos térmicos observados nas curvas TG/DTG e DSC, e os intervalos de temperatura para os sais de potássio estão reunidos na Tabela 3.

Tabela 2. Perdas de massa, atribuídas aos eventos térmicos observados em TG e DSC e intervalos de temperatura para os sais de sódio

\begin{tabular}{|c|c|c|c|c|}
\hline \multirow{2}{*}{ Processo } & \multirow{2}{*}{$\begin{array}{l}\text { Intervalo de Temperatura } \\
\qquad\left({ }^{\circ} \mathrm{C}\right)\end{array}$} & \multicolumn{2}{|c|}{ Perda de Massa ou resíduo* $(\%)$} & \multirow{2}{*}{$\begin{array}{c}\text { Dados }{ }^{\mathrm{a}} \text { de DSC Tempera- } \\
\text { tura de pico }\left({ }^{\circ} \mathrm{C}\right)\end{array}$} \\
\hline & & TG & Calculado & \\
\hline Napyr. $2 \mathrm{H}_{2} \mathrm{O} \rightarrow \mathrm{Napyr}+2 \mathrm{H}_{2} \mathrm{O}$ & $65-140$ & 17,7 & 17,6 & 104,7 (endo) \\
\hline \multirow[t]{2}{*}{ Napyr $\rightarrow$ decomposição } & $250-450$ & 48,1 & 47,8 & 317,6 (exo) \\
\hline & & & & 425,2 (exo) \\
\hline Resíduo para $1 / 2 \mathrm{Na}_{2} \mathrm{SO}_{4}$ & 500 & $34,2 *$ & 34,5 & --- \\
\hline Napip. $2 \mathrm{H}_{2} \mathrm{O} \rightarrow$ Napip $+2 \mathrm{H}_{2} \mathrm{O}$ & $60-140$ & 16,5 & 16,4 & 90,9 (endo) \\
\hline Napip $\rightarrow$ decomposição & $230-350$ & 52,2 & 51,2 & 257,7 (exo) \\
\hline Resíduo para $1 \frac{1}{2} \mathrm{Na}_{2} \mathrm{SO}_{4}$ & 500 & $31,4 *$ & 32,4 & --- \\
\hline Namor. $2,25 \mathrm{H}_{2} \mathrm{O} \rightarrow$ Namor. $0,25 \mathrm{H}_{2} \mathrm{O}+2 \mathrm{H}_{2} \mathrm{O}$ & $60-120$ & 16,6 & 16,0 & 76,3 (endo) \\
\hline Namor. $0,25 \mathrm{H}_{2} \mathrm{O} \rightarrow$ Namor $+0,25 \mathrm{H}_{2} \mathrm{O}$ & $120-150$ & 2,2 & 2,00 & --- \\
\hline \multirow[t]{2}{*}{ Namor $\rightarrow$ decomposição } & $245-400$ & 51,8 & 50,6 & 285,9 (ехо) \\
\hline & & & & 375,5 (ехо) \\
\hline Resíduo para $1 / 2 \mathrm{Na}_{2} \mathrm{SO}_{4}$ & 500 & $29,4 *$ & 31,5 & --- \\
\hline \multirow[t]{2}{*}{ Nahex. $2 \mathrm{H}_{2} \mathrm{O} \rightarrow$ Nahex $+2 \mathrm{H}_{2} \mathrm{O}$} & $50-140$ & 15,5 & 15,4 & 92,2 (endo) \\
\hline & & & & 116,4 (endo) \\
\hline $\operatorname{Nahex}_{(\mathrm{s})} \rightarrow \operatorname{Nahex}_{(\mathrm{l})}$ & $200-220$ & --- & --- & 212,5 (endo) \\
\hline Nahex $_{(1)} \rightarrow$ decomposição & $220-340$ & 54,2 & 54,1 & 280,0 (exo) \\
\hline Resíduo para $1 / 2 \mathrm{Na}_{2} \mathrm{SO}_{4}$ & 500 & $30,3 *$ & 30,4 & --- \\
\hline
\end{tabular}

${ }^{a}$ Exo: processo exotérmico; Endo: processo endotérmico. * equivale a \% de resíduo, os demais são \% para perdas de massa. 


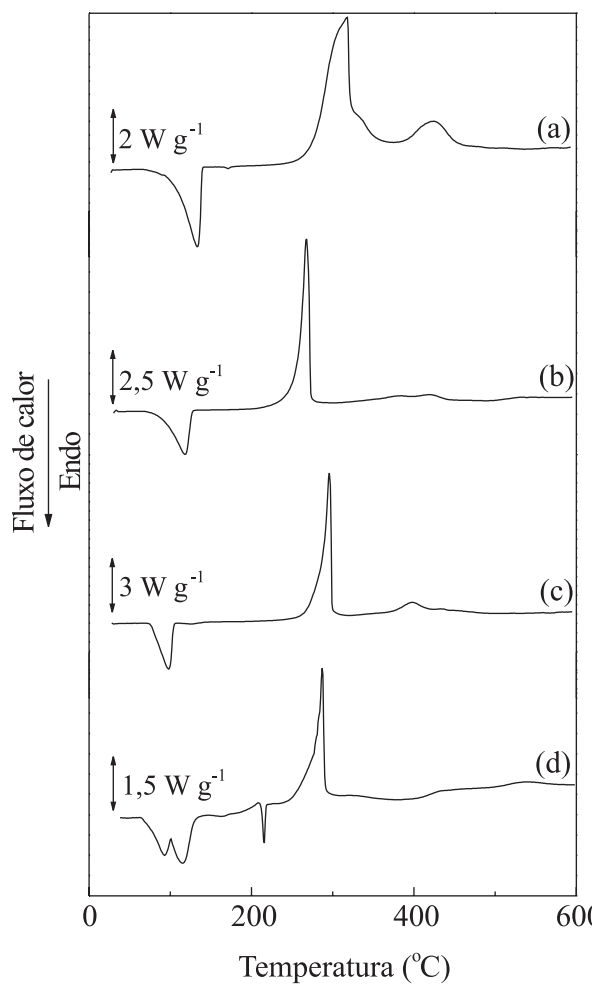

Figura 2. Curvas DSC para os sais: (a) Napyr. $2 \mathrm{H}_{2} \mathrm{O}$; (b) Napip. $2 \mathrm{H}_{2} \mathrm{O}$; (c) Namor. $2 \mathrm{H}_{2} \mathrm{O}$ e (d) Nahex. $2 \mathrm{H}_{2} \mathrm{O}$, sob as condições descritas no texto

As curvas TG/DTG mostram presença de água de hidratação para o Kpip e Kmor e são apresentadas na Figura 3, enquanto as curvas DSC estão na Figura 4.

Após perda da $\mathrm{H}_{2} \mathrm{O}$ de hidratação, os sais apresentaram decomposição em duas etapas. Na primeira etapa ocorre a formação do tiossulfato de potássio $\left(\mathrm{K}_{2} \mathrm{~S}_{2} \mathrm{O}_{3}\right)$ em torno de $300{ }^{\circ} \mathrm{C}$, como pode ser observado na Figura 3. Estes resíduos foram caracterizados por FTIR e concordam com os cálculos estequiométricos. De acordo com a literatura, ${ }^{25} \mathrm{o}$ íon tiossulfato apresenta freqüências de vibração na região de 648, 676, 995, 1120 e $1625 \mathrm{~cm}^{-1}$. Ver espectro de absorção no infravermelho do tiossulfato de potássio e do resíduo no material suplementar Figura 1S.

Em temperaturas mais elevadas este resíduo se converte em

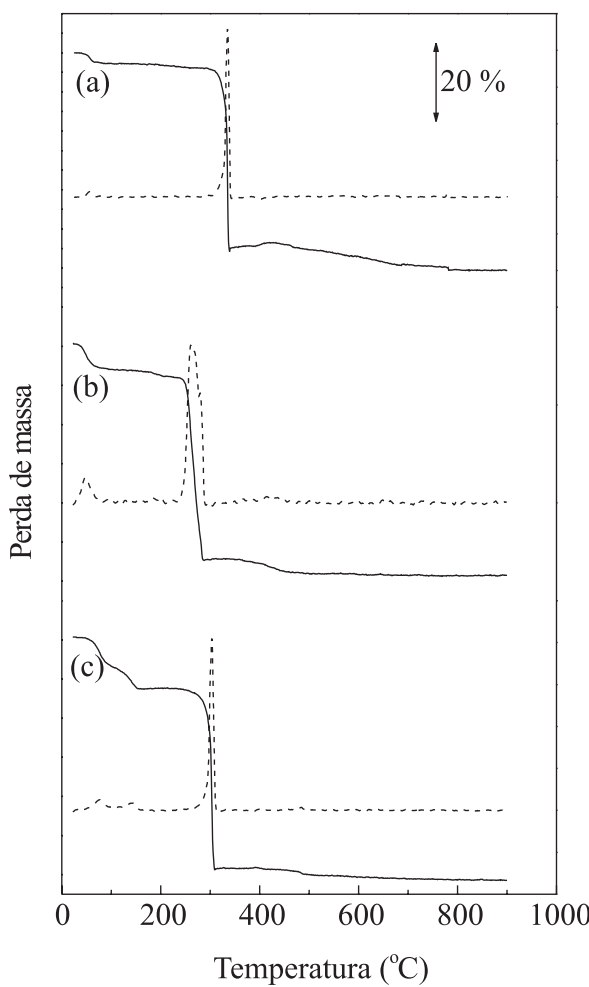

Figura 3. Curvas TG (sólido) e DTG (pontilhado) para os sais: (a) Kpyr; (b) Kpip. $2 \mathrm{H}_{2} \mathrm{O}$; (c) Kmor. $2 \mathrm{H}_{2} \mathrm{O}$, sob as condições descritas no texto

sulfato de potássio, o que pode ser evidenciado pelos cálculos estequiométricos para os resíduos e confirmado por FTIR, em torno de $800{ }^{\circ} \mathrm{C}$. O íon sulfato apresenta bandas na região de 1104 e $623 \mathrm{~cm}^{-1}$, em coincidência com o espectro do $\mathrm{K}_{2} \mathrm{SO}_{4}$. Ver espectro de absorção no infravermelho do sulfato de potássio e do resíduo no material suplementar Figura 2S.

As curvas DSC dos sais de potássio, Figura 4, apresentam picos exotérmicos que concordaram com as curvas TG/DTG nos eventos que envolvem perda de massa, conforme Tabela 3.

\section{CONCLUSÕES}

Os sais de sódio mostraram-se relativamente mais estáveis ter-

Tabela 3. Perdas de massa atribuídas aos eventos térmicos observados em TG e DSC e intervalos de temperatura para os sais de potássio

\begin{tabular}{|c|c|c|c|c|}
\hline \multirow{2}{*}{ Processo } & \multirow{2}{*}{ Intervalo de Temperatura $\left({ }^{\circ} \mathrm{C}\right)$} & \multicolumn{2}{|c|}{ Perda de Massa ou resíduo* (\%) } & \multirow{2}{*}{$\begin{array}{c}\text { Dados }^{\text {a }} \text { de DSC Temperatura de } \\
\text { pico }\left({ }^{\circ} \mathrm{C}\right)\end{array}$} \\
\hline & & TG & Calculado & \\
\hline $\mathrm{Kpyr} \rightarrow 1 / 2 \mathrm{~K}_{2} \mathrm{~S}_{2} \mathrm{O}_{3}$ & $250-350$ & $51,8 *$ & $51,3 *$ & \\
\hline $1 / 2 \mathrm{~K}_{2} \mathrm{~S}_{2} \mathrm{O}_{3} \rightarrow 1 / 2 \mathrm{~K}_{2} \mathrm{SO}_{4}$ & 800 & $46,6^{*}$ & $47,0 *$ & \\
\hline Kpip. $1 \mathrm{H}_{2} \mathrm{O} \rightarrow \mathrm{Kpip}+1 \mathrm{H}_{2} \mathrm{O}$ & $30-200$ & 8,2 & 8,3 & 61,8 (endo) \\
\hline \multirow[t]{2}{*}{$\mathrm{Kpip} \rightarrow 1 / 2 \mathrm{~K}_{2} \mathrm{~S}_{2} \mathrm{O}_{3}$} & $220-300$ & 47,0 & 47,0 & 269,8 (exo) \\
\hline & & & & 418,9 (exo) \\
\hline $1 / 2 \mathrm{~K}_{2} \mathrm{~S}_{2} \mathrm{O}_{3} \rightarrow 1 / 2 \mathrm{~K}_{2} \mathrm{SO}_{4}$ & 700 & $41,4 *$ & 40,1 & --- \\
\hline \multirow[t]{2}{*}{ Kmor. $1 \mathrm{H}_{2} \mathrm{O} \rightarrow \mathrm{Kmor}+1 \mathrm{H}_{2} \mathrm{O}$} & $30-110$ & 7,9 & 7,7 & 79,4 (endo) \\
\hline & $110-170$ & 5,4 & --- & 148,7 (endo) \\
\hline \multirow[t]{2}{*}{ Kmor $\rightarrow 1 / 2 \mathrm{~K}_{2} \mathrm{~S}_{2} \mathrm{O}_{3}$} & $220-400$ & 45,9 & 44,8 & 317,9 (exo) \\
\hline & & & & 350,5 (exo) \\
\hline $1 / 2 \mathrm{~K}_{2} \mathrm{~S}_{2} \mathrm{O}_{3} \rightarrow 1 / 2 \mathrm{~K}_{2} \mathrm{SO}_{4}$ & 700 & $38,6^{*}$ & $37,5^{*}$ & --- \\
\hline
\end{tabular}

${ }^{a}$ Exo: processo exotérmico; Endo: processo endotérmico. * equivale a \% de resíduo, os demais são \% para perdas de massa. 


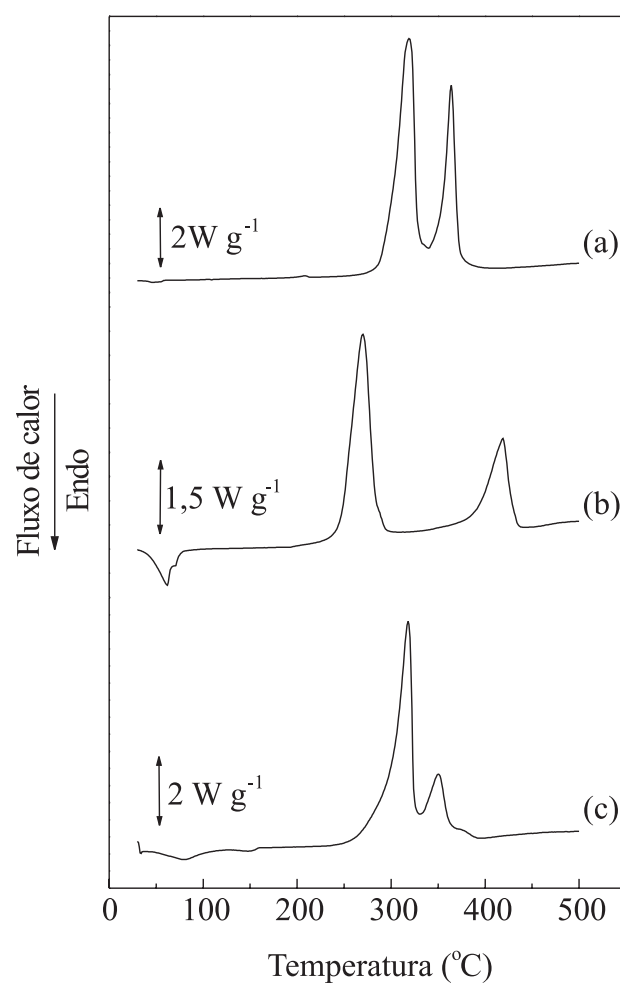

Figura 4. Curvas DSC para os sais: (a) Kpyr; (b) Kpip. $2 \mathrm{H}_{2} \mathrm{O}$; (c) Kmor. $2 \mathrm{H}_{2} \mathrm{O}$, sob as condições descritas no texto

micamente quando comparados com os sais de potássio, do mesmo DTC. Os mecanismos de decomposição térmica dos sais de sódio não seguem o mecanismo proposto por Sharma, ${ }^{26}$ via tiocianato de sódio. O que foi observado é que o mecanismo de decomposição leva a um resíduo de sulfato de sódio em atmosfera oxidante.

Os sais de potássio, por sua vez, também não seguem o mecanismo proposto por Sharma via tiocianato e sim via tiossulfato, que se decompõe a sulfato de potássio em atmosfera oxidante. A ordem de estabilidade após desidratação foi: Napyr $\left(250^{\circ} \mathrm{C}\right) \sim \operatorname{Kpyr}\left(250{ }^{\circ} \mathrm{C}\right)$ $>$ Namor $\left(245^{\circ} \mathrm{C}\right)>\operatorname{Napip}\left(230^{\circ} \mathrm{C}\right)>\operatorname{Kpip}\left(220^{\circ} \mathrm{C}\right) \sim \operatorname{Kmor}(220$ $\left.{ }^{\circ} \mathrm{C}\right)>$ Nahex $\left(200^{\circ} \mathrm{C}\right)$.

\section{MATERIAL SUPLEMENTAR}

No material suplementar, disponível em http://quimicanova.sbq. org.br, com acesso livre, em formato PDF, são apresentados os espectros na região do IV para os resíduos de decomposição do KPyr a $300{ }^{\circ} \mathrm{Ce}$ do tiossulfato de potássio (Figura 1S) e do resíduo do mesmo sal a 800 ${ }^{\circ} \mathrm{C}$ e do sulfato de potássio (Figura $2 \mathrm{~S}$ ), para comparação.

\section{AGRADECIMENTOS}

À FAPESP pelo suporte financeiro (projeto 02/03448-8, 06/06633-1), PROCONTES/USP.

\section{REFERÊNCIAS}

1. Debus, H.; Liebig's Annalen der Chemie 1850, 76, 16, em Thorn, G. D.; Ludwig, R. A.; The dithiocarbamates and related compounds, Elsevier: Amsterdan, 1962.

2. Thorn, G. D.; Ludwig, R. A.; The dithiocarbamates and related compounds, Elsevier: Amsterdam, 1962.

3. Nieuwenhuizen, P. J.; Rubber Chem. Technol. 1997, 70, 368.

4. Marzano, C.; Trevisan, A.; Giovagnini, L.; Fregona, D.; Toxicol. in Vitro 2002, 16, 413.

5. WHO - World Health Organization; Dithiocarbamate pesticides, ethylenethiourea and propylenethyourea: a general introduction, Environmental Health Criteria, 1988, 78, 1.

6. Hill, J. O.; J. Therm. Anal. 1994, 42, 607.

7. Hill, J. O.; Chirawongaram, S.; J. Thermal. Anal. 1994, 41, 511.

8. Sharma, A. K.; Thermochim. Acta 1986, 104, 339.

9. Magee, R. J.; Hill, J. O.; Rev. Anal. Chem. 1985, 8, 5.

10. Sengupta, S. K.; Kumar, S.; Thermochim. Acta 1984, 72, 349.

11. Hill, J. O.; Magee, R. J.; Rev. Inorg. Chem. 1981, 3, 141.

12. Honjo, T.; Imura, H.; Shims, S.; Kiba, T.; Anal. Chem. 1978, 50, 1545.

13. Fabretti, A. C.; Forghieri, F.; Giusti, A.; Preti, C.; Tosi, G.; Spectrochim. Acta, Part A 1984, 40, 343.

14. Cavalheiro, E. T. G.; Ionashiro, M.; Marino, G.; Breviglieri, S. T.; Chierice, G. O.; J. Braz. Chem. Soc. 1999, 10, 65.

15. Polyakov, A. D.; Vasilev, A. N.; Russ. J. Appl. Chem. 1995, 68, 1457.

16. Vasiliev, A. N.; Polackov, A. D.; Molecules 2000, 5, 1014.

17. Ramos, L. A.; Dissertação de Mestrado, Universidade de São Paulo, Brasil, 2003

18. Couto, A. B.; Bernal, C.; Chierice, G. O.; Cavalheiro, E. T. G.; J. Therm. Anal. Cal. 2002, 67, 433 .

19. Antunes, P. A.; Breviglieri, S. T.; Chierice, G. O.; Cavalheiro, E. T. G.; J. Braz. Chem. Soc. 2001, 12, 473.

20. Bernal, C.; Neves, E. A.; Cavalheiro, E.T.G.; Thermochim. Acta 2001, $370,49$.

21. Breviglieri, S. T.; Cavalheiro, E. T. G.; Chierice, G. O.; Thermochim. Acta 2000, 356, 79

22. Cavalheiro, E. T. G.; Ionashiro, M.; Marino, G.; Breviglieri, S. T.; Chierice, G. O.; Transition Met. Chem. 2000, 25, 69.

23. Breviglieri, S. T.; Cavalheiro, E. T. G.; Chierice, G. O.; Polyhedron 1996 , 15,839 .

24. Nakamoto, K.; Infrared and Raman Spectra of Inorganic and Coordination Compounds, $3^{\text {rd }}$ ed.; Wiley: New York, 1978.

25. Miller, F. A.; Wilkins, C. H.; Anal. Chem. 1952, 24, 1253.

26. Sharma, A. K.; Thermochim. Acta 1986, 104, 339. 


\section{ESTUDOS TERMOANALÍTICOS DE SAIS DE DITIOCARBAMATOS DE SÓDIO E POTÁSSIO EM ATMOSFERA OXIDANTE}

Luiz Antônio Ramos, Éder Tadeu Gomes Cavalheiro* e Gilberto Orivaldo Chierice

Instituto de Química de São Carlos, Universidade de São Paulo, Av. do Trabalhador São-Carlense, 400, 13566-950

São Carlos - SP, Brasil

Recebido em 5/12/07; aceito em 11/4/08; publicado na web em

São apresentados os espectros na região do IV para os resíduos de decomposição do KPyr a $300{ }^{\circ} \mathrm{C}$ e do tiossulfato de potássio (Figura $1 \mathrm{~S}$ ) e do resíduo do mesmo sal a $800{ }^{\circ} \mathrm{C}$ e do sulfato de potássio (Figura $2 \mathrm{~S}$ ), para comparação.
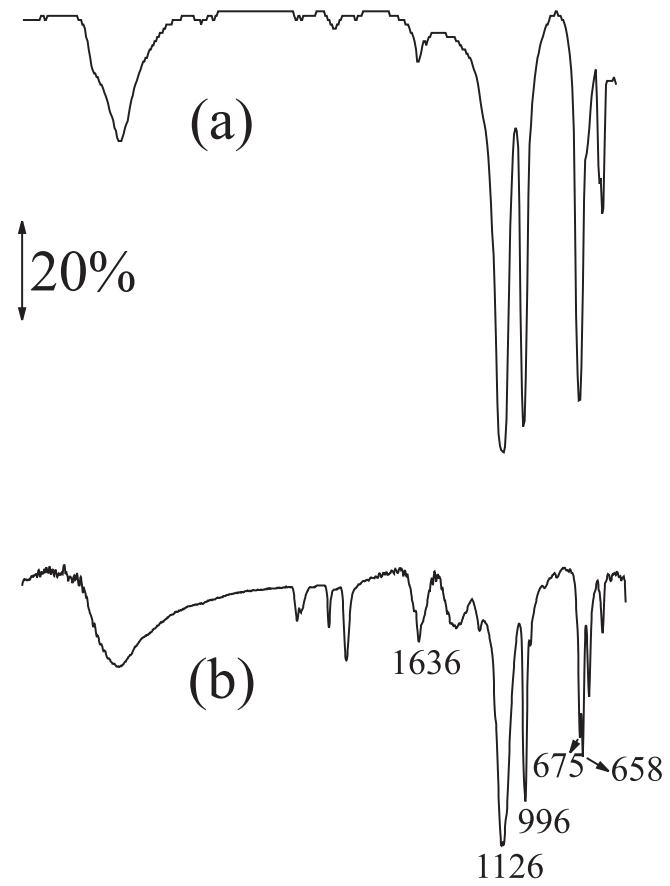

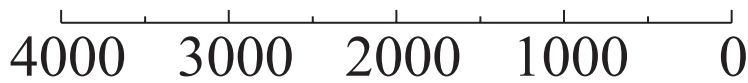 Número de onda $\left(\mathrm{cm}^{-1}\right)$}

Figura 1S. Espectros na região do infravermelho (a) do padrão de tiossulfato de potássio e (b) do resíduo de decomposição do Kpyr coletado a $300{ }^{\circ} \mathrm{C}$
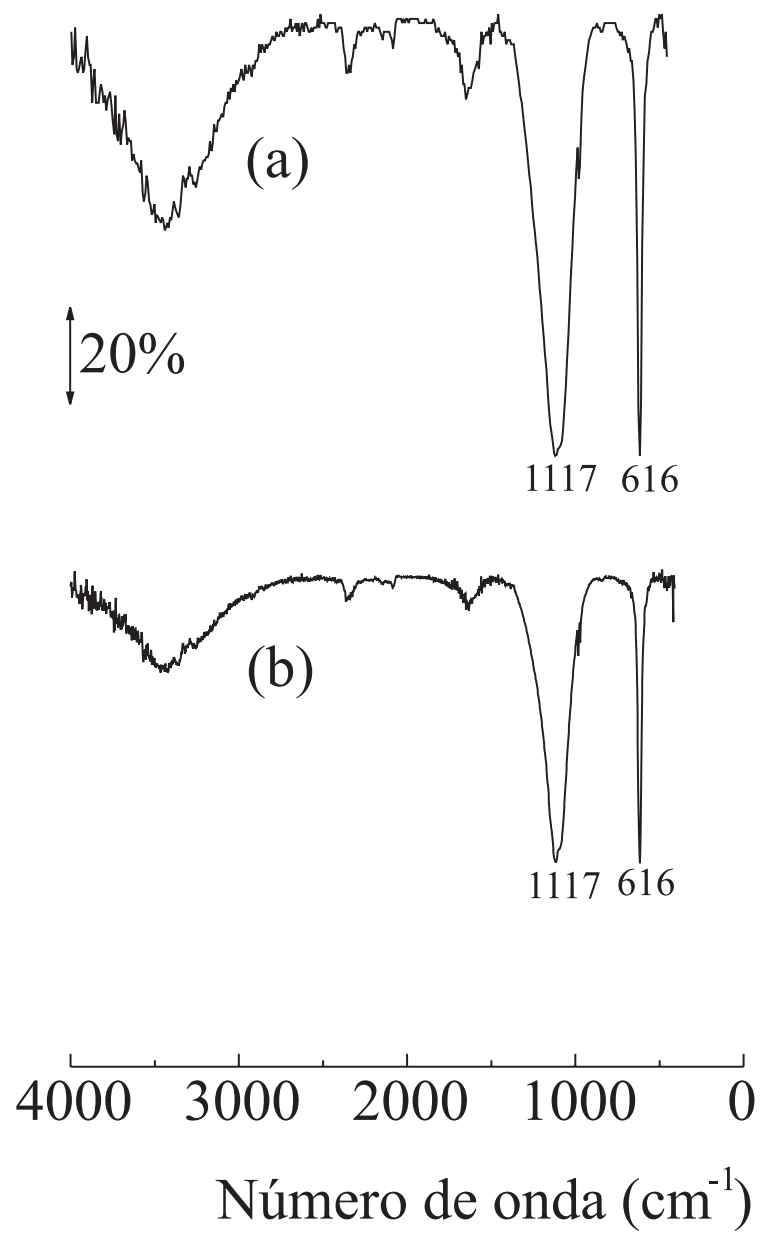

Figura 2S. Espectros na região do infravermelho (a) do padrão de sulfato de potássio e (b) do resíduo de decomposição do Kpyr coletado a $800{ }^{\circ} \mathrm{C}$ 